Review

\title{
A force profile analysis comparison between functional data analysis, statistical parametric mapping and statistical non-parametric mapping in on-water single sculling
} \author{
Todd Pataky ${ }^{g}$ \\ axercise and Sports Science, University of Sydney, Australia \\ ${ }^{\mathrm{b}}$ Physical Education and Sport Sciences, University of Limerick, Ireland \\ ${ }^{c}$ Research Institute for Sport and Exercise Sciences, Liverpool John Moores University, United Kingdom \\ d Department of Rehabilitation Sciences, KU Leuven, Belgium \\ e Department of Mathematics and Statistics, University of Limerick, Ireland \\ f School of Human Sciences (Exercise and Sport Science), University of Western Australia, Australia \\ ${ }^{g}$ Department of Health and Human Sciences, Kyoto University, Japan
}

John Warmenhoven ${ }^{\mathrm{a}, *}$, Andrew Harrison ${ }^{\mathrm{b}}$, Mark A. Robinson ${ }^{\mathrm{c}}$, Jos Vanrenterghem ${ }^{\mathrm{d}}$, Norma Bargary ${ }^{\mathrm{e}}$, Richard Smith ${ }^{\mathrm{a}}$, Stephen Cobley ${ }^{\mathrm{a}}$, Conny Draper ${ }^{\mathrm{a}}$, Cyril Donnelly ${ }^{\mathrm{f}}$,

\section{A R T I C L E I N F O}

\section{Article history:}

Received 27 October 2017

Received in revised form 16 January 2018

Accepted 14 March 2018

Available online $\mathrm{xxx}$

\section{Keywords:}

Statistics

Hypothesis testing

Waveform

Movement

\begin{abstract}
A B S T R A C T
Objectives: To examine whether the Functional Data Analysis (FDA), Statistical Parametric Mapping (SPM) and Statistical non-Parametric Mapping (SnPM) hypothesis testing techniques differ in their ability to draw inferences in the context of a single, simple experimental design.

Design: The sample data used is cross-sectional (two-sample gender comparison) and evaluation of differences between statistical techniques used a combination of descriptive and qualitative assessments. Methods: FDA, SPM and SnPM t-tests were applied to sample data of twenty highly skilled male and female rowers, rowing at 32 strokes per minute in a single scull boat. Statistical differences for gender were assessed by applying two t-tests (one for each side of the boat).

Results: The t-statistic values were identical for all three methods (with the FDA t-statistic presented as an absolute measure). The critical t-statistics $\left(t_{\text {crit }}\right)$ were very similar between the techniques, with SPM $t_{c r i t}$ providing a marginally higher $t_{c r i t}$ than the FDA and SnPM $t_{c r i t}$ values (which were identical). All techniques were successful in identifying consistent sections of the force waveform, where male and female rowers were shown to differ significantly $(p<0.05)$.

Conclusions: This is the first study to show that FDA, SPM and SnPM t-tests provide consistent results when applied to sports biomechanics data. Though the results were similar, selection of one technique over another by applied researchers and practitioners should be based on the underlying parametric assumption of SPM, as well as contextual factors related to the type of waveform data to be analysed and the experimental research question of interest.
\end{abstract}

(c) 2018 Published by Elsevier Ltd on behalf of Sports Medicine Australia.

\section{Introduction}

The ability to statistically analyse whole movements using biomechanical data is of contemporary interest in sport and exercise science. ${ }^{1}$ Movements are often represented by relevant biomechanical time-series variables (also referred to as waveforms, curves, etc.). Differences between individuals for characteristics of

\footnotetext{
* Corresponding author.

E-mail address: jwar9794@uni.sydney.edu.au (J. Warmenhoven).
}

these variables (i.e. the shape of the these curves when observed graphically), has led to terms such as movement 'signatures' being used. ${ }^{2}$ The most commonly used approach for the analysis of these waveform variables however is discrete point analysis (DPA), ${ }^{3}$ which reduces the dimensionality of a waveform by examining pre-selected 'key' data points (e.g. maxima or minima). Despite its common implementation in applied biomechanics settings, this approach can be limiting, as pre-selection of key points is often dependent on a priori knowledge of the movement being analysed, and can thus lead to potentially relevant information being discarded. ${ }^{3}$ This can be become problematic in biomechan- 
ics, as research can often be exploratory prior to the generation of hypotheses when analysing time-series data. ${ }^{4}$

Some well-established statistical methods, which allow statistical examination of entire time-series have increased in popularity in human movement research. These are Functional Data Analysis (FDA) ${ }^{5}$ and Statistical Parametric Mapping (SPM). ${ }^{6}$ The general concept of FDA is to express discrete observations arising from time-series in the form of a function, and then consider each measured function as a single observation for subsequent statistical analysis. This has led to the adaptation of several accepted statistical practices that are commonly used for data reduction (i.e. PCA), clustering, hypothesis testing techniques (i.e. functional linear models) and forecasting (for a comprehensive contemporary review see: Ullah \& Finch ${ }^{7}$ ). Within the realm of human movement analysis, FDA has been applied in a range of sports biomechanics studies including, but not limited to, the analysis of race-walking, running, jumping, weightlifting and rowing. ${ }^{8-14}$ SPM also regards time-series variables as a single observation. SPM exploits the use of random field theory (RFT $)^{15}$ to directly map the conventional Gaussian distribution to smooth $n$-dimensional continua, providing an objective framework for hypothesis testing using parametric statistical concepts. SPM has also been adapted for non-parametric analyses, ${ }^{16}$ and this is referred to as Statistical nonParametric Mapping (SnPM). Similar to FDA, SPM (and SnPM) have demonstrated application in human movement contexts such as the analysis of biomechanical time-series data in soccer kicking, running and cutting movements and landing techniques. ${ }^{17-21}$

The recent proliferation of these statistical methods in biomechanics is partly due to their increasing availability. Software for the implementation of FDA and SPM is freely available and written for use with a number of statistical programs. FDA software is available from the www.functionaldata.org website, and is designed for use with Matlab, R and S-Plus. Similarly, SPM software is available for use from the www.spm1d.org website, and is designed for use with Matlab and Python. Despite being different statistical methods for analysing waveform data, there are some techniques within FDA and SPM that mirror conventional statistical concepts from classical hypothesis testing. One such statistical test is the independent samples t-test. In both FDA and SPM, a t-statistic is generated in the form of a continuous trajectory and random data is used to develop a critical t-statistic threshold for significance testing between independent samples. At present, the independent sample t-test has been applied in the context of FDA, ${ }^{22} \mathrm{SPM}^{23}$ and also SnPM ${ }^{24}$ with biomechanical data. Despite this, to the best of the authors' knowledge there has never been a formal comparison of these statistical approaches in the context of human movement data.

This paper examines the FDA, SPM and SnPM independent sample t-tests when applied to sample rowing biomechanics data, to test the hypothesis of gender differences in sculling. Understanding gender differences in rowing has practical relevance in sports biomechanics, as it can provide insights into the need for gender specific training interventions and evaluation of injury mechanisms. At present there is established support for presence of biomechanical differences between male and female rowers for discrete biomechanical variables, with peak force and peak power differences noted between males and females. ${ }^{25}$ Additionally, patterns of relative force-angle profiles (normalized to $100 \%$ of maximum force) have also been found to differ relative to gender. ${ }^{14}$ Thus it can be hypothesised from these previous findings that differences between these samples of rowers may exist in the amount of force application applied across sections of the drive phase, however this is largely an exploratory analysis using each of the statistical approaches. The goal was to qualitatively compare the results of FDA, SPM and SnPM for an example dataset, and subse- quently to describe factors specific to each technique which may yield different outcomes for arbitrary datasets.

\section{Methods}

Following institutional ethical approval and participant consent, ten male $(M$ age $=21.32+2.00$ years; $M$ height $=1.92+0.06 \mathrm{~m}$; $M$ mass $=86.99+9.19 \mathrm{~kg})$ and ten female $(F$ age $=20.28+3.17$ years; $F$ height $=1.84+0.04 \mathrm{~m} ; F$ mass $=75.37+6.36 \mathrm{~kg}$ ) highly trained heavyweight and lightweight scullers participated. All rowers were required to have competed in Australian national age group or open competitions prior to testing (i.e. national level athlete), or represented Australia at an under 18, 23, or open level event (i.e. international level athlete) prior to testing.

Participants were instructed to row a total of $1000 \mathrm{~m}$, composed of four $250 \mathrm{~m}$ segments at ascending pre-selected stroke rates of 20 , 24,28 and 32 strokes per minute. Only the 32 strokes per minute data (i.e., a race representative stroke rate) were analysed. Rowing data was obtained using ROWSYS instrumentation (see Smith and Loschner ${ }^{26}$ for a full description of the system), and the propulsive component (relative to the longitudinal axis of the boat) of force was retained and analysed. The same ten strokes were selected for the bow and stroke side for each rower (these sides of the boat are sometimes also referred to as starboard and port-side). These strokes were performed simultaneously and consecutively. For each participant, the drive and recovery phases were identified using the horizontal oar angle and only the drive phase was analysed in this study. A linear length normalization strategy using an interpolating cubic spline was applied, registering each curve to $100 \%$ of the drive phase. An average waveform created from each participant's ten strokes (for both boat-sides) was used for further analysis. This resulted in a total of twenty curves for each independent samples t-test. Prior to analysis, data was filtered using a dual low-pass Butterworth filter with a cut-off frequency of $5 \mathrm{~Hz}$.

For the FDA, SPM and SnPM t-tests $\alpha=0.05$. Prior to conducting the FDA independent $t$-test, the force trajectories were estimated as functions using B-splines. ${ }^{5}$ Data was also smoothed as a part of FDA by adding a roughness penalty to the fitting procedure. A very small roughness penalty was used $\left(1 \times 10^{-100}\right)$ to avoid introducing any differences between FDA, SPM and SnPM that were a part of data pre-processing. For FDA, SPM and SnPM a t-statistic trajectory was created across the entire movement using the following two equations. The FDA t-statistic [FDA T(t)] was calculated as ${ }^{27,28}$ :

$$
\operatorname{FDAT}(t)=\frac{\left|\bar{x}_{1}(t)-\bar{x}_{2}(t)\right|}{\sqrt{\frac{1}{n_{1}} \operatorname{Var}\left[x_{1}(t)\right]+\frac{1}{n_{2}} \operatorname{Var}\left[x_{2}(t)\right]}}
$$

where, $x_{1}(t)$ and $x_{2}(t)$ are the force trajectories for males and females and $\bar{x}_{1}(t)$ and $\bar{x}_{2}(t)$ are the pointwise means for the male and female trajectories, $\mathrm{n} 1$ and $\mathrm{n} 2$ denote the sample sizes in male and female trajectories respectively and $\operatorname{Var}\left[x_{1}(t)\right]$ and $\operatorname{Var}\left[x_{2}(t)\right]$ denote the variance for male and female trajectories respectively. The pointwise t-statistic for both SPM and SnPM [SPM \& SnPM $\mathrm{T}(t)]$ is identical (equation below), however it is directional as the numerator of $T(t)$ is not absolute (similar to Welch's original definition of the conventional t-statistic):

$$
\operatorname{SPM} \& \operatorname{SnPMT}(t)=\frac{\bar{x}_{1}(t)-\bar{x}_{2}(t)}{\sqrt{\frac{1}{n_{1}} \operatorname{Var}\left[x_{1}(t)\right]+\frac{1}{n_{2}} \operatorname{Var}\left[x_{2}(t)\right]}}
$$

Calculation of the critical t-statistic for the FDA t-test (FDA- $t_{\text {crit }}$ ) was determined using a permutation test by randomly shuffling the male and female labels on the trajectories and calculating the maximum of $T(t)$ using these new labels. ${ }^{28}$ The maximum number of permutations possible for this data was used to create a null distribution $(184,756$ permutations or $20 ! /(10 ! \times 10 !))$. For 
each permutation the maximum t-value $\left(t_{\max }\right)$ is saved, resulting in a distribution of $t_{\max }$ values. The FDA- $t_{\text {crit }}$ is then given as the $100 \times(1-\alpha)^{\text {th }}$ percentile of the $t_{\max }$ distribution. The critical t-statistic for the SPM t-test (SPM- $t_{\text {crit }}$ ) is given by $\mathrm{RFT}^{15}$ as the solution to:

$P\left(T(t)_{\max }>t_{\text {crit }}\right)=1-\exp \left(-\int_{t_{\text {crit }}}^{\infty} f_{p d f}(x) d x-E D\right)=\alpha$

where $T(t)_{\max }$ is the maximum value of the continuous trajectory, $t_{c r i t}$ is the SPM- $t_{c r i t}, f_{p d f}(x)$ is the t-statistic's probability density function and $E D$ is the smoothness-dependent Euler density function. ${ }^{6}$ Similar to the conventional t-test, the above equation represents the probability that $T(t)_{\max }$ exceeds SPM- $t_{\text {crit }}$ when the underlying data refers directly to random processes with Gaussian kernel. ${ }^{24}$ Identically to the FDA- $t_{c r i t}$, the t-statistic for the SnPM t-test $\left(\mathrm{SnPM}-\mathrm{t}_{\text {crit }}\right)$ is calculated using the permutation method implemented by Nichols and Holmes ${ }^{16}$ and is summarized comprehensively in Pataky et al. ${ }^{24}$ Again, the maximum number of permutations possible for this data was used to create a null distribution (this was 184,756 permutations).

FDA, SPM and SnPM t-tests were conducted independently, for each side of the boat to test for gender differences in the drive phase (continuous application of propulsive force). For each t-test, the critical t-statistic and areas of significance between the two groups were reported. Descriptive comparisons of the critical t-statistic thresholds for the FDA, SPM and SnPM t-tests and any associated areas of significance (or regions) was also used as the criterion for comparing each of the statistical techniques.

\section{Results}

For the bow-side, the FDA- $t_{c r i t}=3.20$, the SPM- $t_{c r i t}=3.33$ and the SnPM- $t_{\text {crit }}=3.20$. FDA, SPM and SnPM t-tests each identified two separate parts of the drive phase that differed significantly between males and females $(\alpha=0.05)$. Firstly, a region spreading from $28 \%-82 \%$ of the drive phase was found to differ significantly for gender, with each method (FDA $p<0.001$; SPM $p<0.001$; SnPM $p<0.001)$. Secondly, a region spreading from $90 \%-96 \%$ of the drive phase was found to differ significantly for gender with each method (FDA $p<0.001$; SPM $p=0.040$; SnPM $p<0.001$ ).

For the stroke side, the FDA- $t_{\text {crit }}=3.18$, the SPM- $t_{\text {crit }}=3.33$ and the SnPM- $t_{\text {crit }}=3.18$. FDA, SPM and SnPM t-tests each identified one part of the drive phase that significantly differed between males and females $(\alpha=0.05)$. This region spread from $26 \%-82 \%$ of the drive phase was found to significantly differ for gender with each method (FDA $p<0.001$; SPM $p<0.001$; SnPM $p<0.001$ ). For the bow-side and stroke-side comparisons, the t-statistic trajectories for the FDA, SPM and SnPM methods can be seen in Fig. 1.

\section{Discussion}

This study compared the results of the FDA, SPM and SnPM t-tests in the identification of differences in propulsive force application for gender using a sample rowing data set. All three techniques provided similar results, and in each case significant differences were identified for gender, with significantly different sections of the force curve present across both sides of the boat. These sections were identical for each t-test. All three t-tests demonstrated that male rowers were significantly more likely to apply a higher level of force leading into and away from the point of maximum force on both sides of the boat. As anticipated, these results are consistent with previous findings related to kinetic differences between male and female rowers. ${ }^{25}$
From a statistical perspective, there are several noteworthy findings for future application of these techniques in biomechanics. The t-statistics calculated as a part of each approach were numerically identical, with the exception that the SPM and SnPM t-statistics are directional, and thus provide a potentially more informative graph for inspection of differences between independent groups. Of particular note, the SPM Matlab function ttest2 and the SnPM Matlab function nonparam.ttest2 allow the possibility for conducting either a one-tailed or two-tailed t-test, whereas the tperm_fd Matlab function for the FDA t-test only provides a two-tailed option. This is something that should be considered when setting the alpha level for future use of the FDA technique.

The FDA- $t_{\text {crit }}$, SPM- $t_{\text {crit }}$ and SnPM- $t_{\text {crit }}$ values were very similar (with the FDA- $t_{\text {crit }}$ and the SnPM- $t_{\text {crit }}$ providing identical values). This led to global consistency between the techniques in identifying of statistically significant sections of difference between genders for their respective force curves. Two sections of the curve were identified for the bow-side and one section of the curve was identified for the stroke-side. The small differences in $t_{\text {crit }}$ values between each of these techniques is partly due to the different processes implemented for the generation of the null distribution. Generally speaking, parametric hypothesis testing techniques (e.g. cross-sectional, longitudinal, regression, etc.), are typically considered to be a part of the general linear model (GLM) framework. SPM applies the GLM framework to $n$-dimensional data, ${ }^{29,30}$ by fitting a GLM at each data point in a time-series, followed by parametric inference and corrections for multiple comparisons via RFT. ${ }^{6}$ Such parametric approaches assume that the residual trajectories are normally distributed. Conversely, permutation tests such as the FDA and SnPM t-tests in the present study are nonparametric and rely on the less inclusive assumption of exchangeability: under the permutation-test null hypothesis. ${ }^{31}$ In the present study, both groups of curves are believed to be generated by a single distribution, where the independent and identically distributed observations are exchangeable. It is entirely plausible that these different (parametric vs non-parametric) approaches for generation of the null distribution, could have led to the small difference noted between the $t_{\text {crit }}$ for SPM and the $t_{\text {crit }}$ for non-parametric tests (FDA and $\mathrm{SnPM}$ ). When explored in the context of imaging data, permutation tests have been demonstrated to be generally more stringent than parametric tests (i.e. stronger control over Type- 1 errors) and more robust to random noise in imaging measurements. ${ }^{31}$ The results from the present study are somewhat opposed to these findings, where the SPM- $t_{c r i t}$ was higher than the $t_{c r i t}$ for the permutation tests. There are some potential reasons for this finding. Firstly, parametric approaches such as SPM make assumptions regarding the presence of normality. ${ }^{16}$ Such strategies can become prone to false findings when this underlying assumption is invalid. It is possible that this may have influenced results in the present study. Additionally, small sample sizes like that noted in the present study (10 trajectories per sample) can lead to insufficiently small numbers of permutations for building a null distribution. This can also potentially affect calculation of $t_{\text {crit }}$ for the permutation tests, although this was unlikely to have a strong affect in the present study as a large number of permutations were used (over 100,000). Given the similarity of all critical t-statistic thresholds, the choice between parametric (SPM) and non-parametric (FDA and SnPM) t-tests in the present study had negligible effects on the current results suggesting that RFT's assumption of waveform based Gaussian randomness was reasonable for the current data set, with this also previously demonstrated to be true with other biomechanical data. ${ }^{24}$ Similarly, there were no notable differences between the functional approach used in the FDA t-test (representing the data as coefficients of equations), and the SPM and SnPM methods (where in the present study the data was represented as a vector of points). Further to this, the permutation approaches outlined 

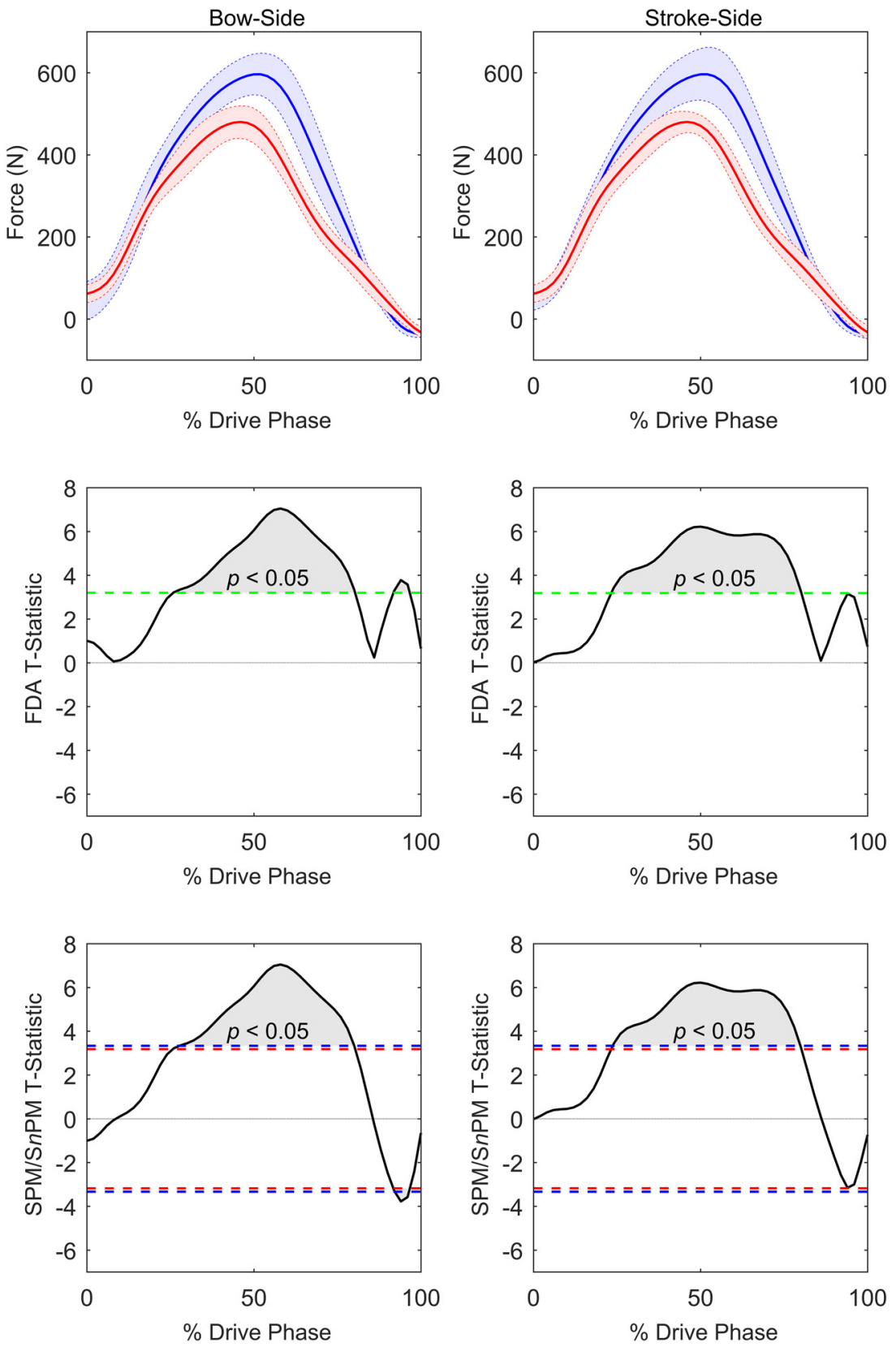

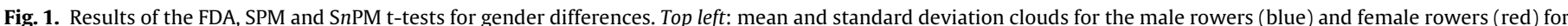

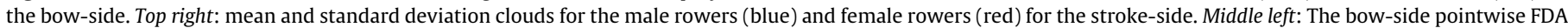

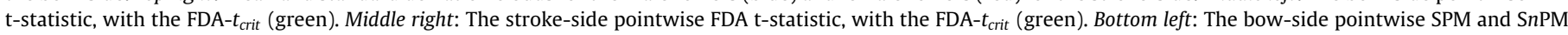

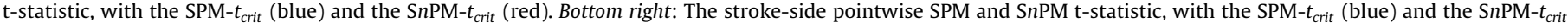
(red). (For interpretation of the references to colour in this figure legend, the reader is referred to the web version of this article.)

for the FDA t-test and the SnPM t-test are theoretically identical, with the exception of how the data is represented prior to each t-test being conducted (i.e. functional data opposed to a vector or points). To the best of the authors' knowledge, this is the first paper to demonstrate theoretical statistical equivalence between these two non-parametric approaches. Additionally, this paper indicates that when smoothing is controlled for during FDA, no registration techniques are applied for temporal misalignments of data post normalization, ${ }^{5}$ and parametric assumptions underpinning the data hold true, FDA, SPM and SnPM results are very similar.

In light of these results it could be asked whether there is an optimal statistical approach amongst FDA, SPM and SnPM when applying the independent samples t-test? In short, the answer is that there is no optimal method. There are some reasons however, why researchers and practitioners may opt to select one method over another for hypothesis testing of waveform data. Firstly, and as outlined by Pataky et al., ${ }^{24}$ the main benefit of SPM(and RFT) tests is that, since they assume an analytical model of randomness and they are computationally fast. Conversely, non-parametric procedures are computationally slower because they build randomness models iteratively. Similar to conventional parametric hypothesis testing approaches, a substantial issue for their application is the violation of the assumption for a Gaussian distribution of randomness. Pataky et al. ${ }^{24}$ have noted that adherence to the normality assumption should be assessed prior to using parametric procedures, either explicitly through a test for normality, or implicitly by checking for agreement between parametric and non-parametric results. At present, methods adapted for testing normality of wave- 
form data in biomechanical literature is limited, however, some methods have been suggested and used in imaging research, ${ }^{32}$ with this being a potential area of exploratory interest in human movement research. Additionally, the FDA and SnPM permutation approaches in the present study are less susceptible to spurious results when smaller sample sizes are used, and may be of benefit in those contexts in biomechanical research. ${ }^{24}$

Some caution is advised however for applying the FDA and SnPM approaches in future research. As both of these are non-parametric permutation methods, they rely upon the user to designate a suitable number of permutations to build the null distribution. If the maximum number of permutations is not selected, this will lead to the generation of a different critical t-threshold every time the test is re-run using the same data. The authors advise that if possible, future use of these techniques should aim to use the maximum number of permutations possible (or use a suitable large minimum number of permutations, i.e. 100,000). Unlike FDA and SnPM, the SPM critical t-threshold will always be stable. Additionally, for the non-parametric FDA t-test it should also be noted that a negligible smoothing parameter was added to the data as a part of fitting Bsplines to the trajectories. It should be cautioned that the addition of a smoothing parameter to previously filtered data is unnecessary, and should be avoided in future experimental research. A smoothing parameter was added in the present study as the MAT$\mathrm{LAB}$ software requires the integration of a smoothing parameter as a part of the function fitting process.

Each statistical method also allows for some unique benefits, which may suit researchers depending upon the context of their research question and also the type of data involved in the study. FDA and SPM are useful for smooth waveform data, however FDA provides researchers with control over the amount and type of smoothing, registration of functions to separate amplitude and phase variation, and also allows for accurate calculation of derivatives, which can be more effective for registering functions in some instances. ${ }^{5}$ It should also be mentioned that FDA techniques would generally not be applied using the method outlined in the present study. Despite control over smoothing being integral to the comparison of FDA and SPM in this study, smoothing is considered to be an integral feature of FDA, and the extent to which data are smoothed normally involves careful consideration and pre-selection using approaches such as generalized cross validation (GCV). ${ }^{5}$

There may also be occasions where SPM and SnPM are considered as more suitable alternatives to FDA, with one such example being when a variable has important temporal variations in frequency content, thus making it difficult for a single basis expansion approach (B-splines, Fourier, wavelets, etc.) to adequately represent the original data as coefficients of an equation (i.e. in the example of running gait, high frequency content in ground reaction force at foot-strike and then lower frequency content throughout the rest of the stance phase). Additionally, as SPM and SnPM treats data as a vector of points, these approaches are more flexible for a priori selection of important regions of interest (ROI) on a waveform, ${ }^{33}$ and allows for hypothesis testing to take place on these ROIs (thus influencing statistical power), rather than the whole vector. This is not possible within FDA. As a cautionary point for the potential user of SPM or SnPM however, choosing smaller regions within a waveform will change the critical t-threshold for a given alpha level (as the smoothness of the curve will change, thus influencing the outcomes of RFT). Researchers, therefore, must have a strong rationale for pre-selection of a section of a movement to be analysed, as the results of an ROI analysis will likely change, when compared to SPM or SnPM being applied to the entire time-series. ${ }^{33}$

Finally, it should also be acknowledged that there are advancements in FDA beyond the scope of the FDA technique applied in the present study. ${ }^{34}$ From the perspective of FDA hypothesis testing techniques it appears that there are two main approaches (parametric and non-parametric), which fall within basis function approximation methods and overall tests. With reference to procedures concerned with testing the equality of coefficients from a basis function approximation, parametric methods include the works of Fan and $\mathrm{Li}^{35}$ Cuevas et al., ${ }^{36}$ and Spitzner ${ }^{37}$; and nonparametric methods include the work of Zhang and Chen, ${ }^{38}$ Delgado, ${ }^{39}$ and Cao et al. ${ }^{40}$ The FDA t-test used in the present study $^{28}$ was explored due to its implementation with biomechanical data in experimental human movement research, and also the ease with which software can be accessed by applied clinicians and researchers from the FDA website.

\section{Conclusion}

The FDA, SPM and SnPM statistical methods all came to the same inferential conclusions (t-test) using this sample data set, despite possessing alternative approaches for representation of the raw time-series data and estimation of the null distribution. As such, it is likely that selection of one technique over another will likely be due to the type of data being used and the nature of the experimental research question involved. This is also the first study to demonstrate statistical equivalence of the FDA t-test and SnPM t-tests.

\section{Practical implications}

- The SPM t-test is suited for application to parametric distributions of curves, and may also be useful when it is desirable to explore key ROIs on a waveform, or manage a signal with high and low frequency content.

- The SnPM t-test is suited for application to non-parametric distributions of curves, and like SPM, may be useful when it is desirable to explore key ROIs on a waveform, or manage a signal with high and low frequency content.

- The FDA t-test is suited for application to non-parametric distributions of curves, and is practically useful when fine control over the smoothing and registration (i.e. separation of phase and amplitude variation and temporal alignment) of data is of interest for particular experimental research questions.

**These points pertain to current software implementations for each of these techniques. Modifications to these software packages may lead to changes in the benefits of some of these techniques in the future.

\section{Acknowledgements}

The authors would like to acknowledge an International Society of Biomechanics in Sport for an Early Career Researcher Grant that was used to support the completion of this study. There were no additional sources of funding.

\section{References}

1. Preatoni E, Hamill J, Harrison AJ et al. Movement variability and skills monitoring in sports. Sports Biomech 2013; 12(2):69-92.

2. Nimphius S, McGuigan MR, Suchomel TJ et al. Variability of a force signature during windmill softball pitching and relationship between discrete force variables and pitch velocity. Hum Mov Sci 2016; 47:151-158.

3. Richter C, Marshall B, Moran K. Comparison of discrete-point vs. dimensionalityreduction techniques for describing performance-related aspects of maximal vertical jumping. J of Biomech 2014; 47(12):3012-3017.

4. Pataky TC, Vanrenterghem J, Robinson MA. The probability of false positives in zero-dimensional analyses of one-dimensional kinematic, force and EMG trajectories. J Biomech 2016; 49(9):1468-1476.

5. Ramsay JO, Silverman BW. Functional Data Analysis, Wiley Online Library, 2005.

6. Friston KJ, Ashburner JT, Kiebel SJ et al. Statistical Parametric Mapping: The Analysis of Functional Brain Images, Amsterdam, Elselvier/Academic press, 2007.

7. Ullah S, Finch CF. Applications of functional data analysis: a systematic review. BMC Med Res Methodol 2013; 13(1):43. 
8. Dona G, Preatoni E, Cobelli C et al. Application of functional principal component analysis in race walking: an emerging methodology. Sports Biomech 2009; 8(4):284-301.

9. Donoghue OA, Harrison AJ, Coffey $\mathrm{N}$ et al. Functional data analysis of running kinematics in chronic Achilles tendon injury. Med Sci Sports Exerc 2008; 40(7):1323-1335.

10. Harrison A, Ryan W, Hayes K. Functional data analysis of joint coordination in the development of vertical jump performance. Sports Biomech 2007; 6(2):199-214.

11. Kipp K, Redden J, Sabick M et al. Kinematic and kinetic synergies of the lower extremities during the pull in Olympic weightlifting. J Appl Biomech 2012; 28(3):271-278.

12. Kipp K, Redden J, Sabick MB et al. Weightlifting performance is related to kinematic and kinetic patterns of the hip and knee joints. J Strength Cond Res 2012; 26(7):1838-1844.

13. Warmenhoven J, Cobley S, Draper C et al. Assessment of propulsive pin force and oar angle time-series using functional data analysis in on-water rowing. Scand J Med Sci Sports 2017; 27(12):1688-1696.

14. Warmenhoven J, Cobley S, Draper C et al. How gender and boat-side affect shape characteristics of force-angle profiles in single sculling: insights from functional data analysis. J Sci Med Sport 2018; 21(5):533-537.

15. Adler R, Taylor J. Random Fields and Geometry, Springer New York, Springer Monographs in Mathematics, 2007.

16. Nichols TE, Holmes AP. Nonparametric permutation tests for functional neuroimaging: a primer with examples. Hum Brain Mapp 2002; 15(1):1-25.

17. Augustus S, Mundy P, Smith N. Support leg action can contribute to maximal instep soccer kick performance: an intervention study. J Sports Sci 2017; 35(1):89-98.

18. David S, Komnik I, Peters M et al. Identification and risk estimation of movement strategies during cutting maneuvers. J Sci Med Sport 2017; 20(12):1075-1080.

19. Fox A, Bonacci J, McLean S et al. Efficacy of ACL injury risk screening methods in identifying high-risk landing patterns during a sport-specific task. Scand J Med Sci Sports 2017; 27(5):525-534.

20. Vanrenterghem J, Venables E, Pataky T et al. The effect of running speed on knee mechanical loading in females during side cutting. J Biomech 2012; 45(14):2444-2449.

21. Whyte EF, Richter C, O'Connor S et al. The effect of high intensity exercise and anticipation on trunk and lower limb biomechanics during a crossover cutting manoeuvre. J Sports Sci 2017:1-12.

22. Baumgart C, Hoppe MW, Freiwald J. Phase-specific ground reaction force analyses of bilateral and unilateral jumps in patients with ACL reconstruction. Orthop J Sports Med 2017; 5(6), 2325967117710912.

23. Schuermans J, Danneels L, Van Tiggelen D et al. Proximal neuromuscular control protects against hamstring injuries in male soccer players: a prospective study with electromyography time-series analysis during maximal sprinting. Am J Sports Med 2017; 45(6):1315-1325.
24. Pataky TC, Vanrenterghem J, Robinson MA. Zero-vs one-dimensional, parametric vs. non-parametric, and confidence interval vs. hypothesis testing procedures in one-dimensional biomechanical trajectory analysis. J Biomech 2015; 48(7):1277-1285.

25. McGregor A, Patankar Z, Bull A. Do men and women row differently? A spinal kinematic and force perspective. Proc Inst Mech Eng P J Sports Eng 2008 222(2):77-83.

26. Smith RM, Loschner C. Biomechanics feedback for rowing. J Sports Sci 2002; 20(10):783-791.

27. Coffey N, Hinde J. Analyzing time-course microarray data using functional data analysis-a review. Stat Appl Genet Molec Biol 2011; 10(1).

28. Ramsay JO, Hooker G, Graves S. Functional Data Analysis with R and MATLAB, Springer Science \& Business Media, 2009.

29. Ashburner J, Friston KJ. Voxel-based morphometry-the methods. NeuroImage $2000 ; 11(6): 805-821$.

30. Friston KJ, Holmes AP, Worsley KJ et al. Statistical parametric maps in functional imaging: a general linear approach. Hum Brain Mapp 1995; 2(4):189-210.

31. Awate SP, Yushkevich PA, Song Z et al. Cerebral cortical folding analysis with multivariate modeling and testing: studies on gender differences and neonatal development. NeuroImage 2010; 53(2):450-459.

32. Luo W-L, Nichols TE. Diagnosis and exploration of massively univariate neuroimaging models. Neurolmage 2003; 19(3):1014-1032.

33. Pataky TC, Robinson MA, Vanrenterghem J. Region-of-interest analyses of onedimensional biomechanical trajectories: bridging $0 \mathrm{D}$ and $1 \mathrm{D}$ theory, augmenting statistical power. PeerJ 2016; 4, e2652.

34. Corain L, Melas VB, Pepelyshev A et al. New insights on permutation approach for hypothesis testing on functional data. Adv Data Anal Classif 2014; 8(3):339-356.

35. Fan J, Lin S-K. Test of significance when data are curves. J Am Stat Assoc 1998 93(443):1007-1021.

36. Cuevas A, Febrero M, Fraiman R. An anova test for functional data. Comput Stat Data Anal 2004; 47(1):111-122.

37. Spitzner DJ. A powerful test based on tapering for use in functional data analysis. Electron J Stat 2008; 2:939-962.

38. Zhang J-T, Chen J. Statistical inferences for functional data. Ann Stat 2007; 35(3):1052-1079.

39. Delgado MA. Testing the equality of nonparametric regression curves. Stat Probabil Lett 1993; 17(3):199-204.

40. Cao G, Yang L, Todem D. Simultaneous inference for the mean function based on dense functional data. J Nonparametr Stat 2012; 24(2):359-377. 